\title{
ESTRUTURA VEGETAL E GRAU DE PERTURBAÇÃO DOS MANGUEZAIS DA LAGOA DA TIJUCA, RIO DE JANEIRO, RJ, BRASIL
}

\author{
SOARES, M. L. G. \\ Universidade do Estado do Rio de Janeiro, Departamento de Oceanografia, \\ Rua São Francisco Xavier, 524, 4º andar, CEP 20550-013, Rio de Janeiro, RJ \\ Correspondência para: Mário Luiz Gomes Soares, Universidade do Estado do Rio de Janeiro, \\ Departamento de Oceanografia, Rua São Francisco Xavier, 524, 4o andar, CEP 20550-013, \\ Rio de Janeiro, RJ, e-mail: mariolgs@uerj.br \\ Recebido em 14/11/97 - Aceito em 04/09/98 - Distribuído em 10/09/99
}

(Com 2 figuras)

\section{ABSTRACT \\ Structure and degree of perturbation of mangroves at Tijuca Lagoon, Rio de Janeiro, RJ, Brazil}

The Tijuca Lagoon is located south of Rio de Janeiro City. It is bordered by a discontinued and narrow belt of mangrove trees. Mangrove forests have a high structural variability. Of the eighteen stands studied there are monospecifics stands of Laguncularia racemosa with dbh between 3.4 and $7.7 \mathrm{~cm}$ and height between 4.5 and $7.7 \mathrm{~m}$, dominated by trees with dbh less than $10.0 \mathrm{~cm}$. At the other stands, occur mixed forests, structuraly more developed, sometimes dominated by Laguncularia racemosa, sometimes by Rhizophora mangle (dbh between 7.8 and $16.7 \mathrm{~cm}$; height between 6.8 and $16.1 \mathrm{~m}$, prevailing trees with dbh greater than $10.0 \mathrm{~cm}$ ). There are also mixed stands dominated by Avicennia schaueriana (dbh of $7.1 \mathrm{~cm}$ and $5.3 \mathrm{~m}$ high, predominating individuals over $10.0 \mathrm{~cm}$ in $\mathrm{dbh}$ ). The structural variability observed, is strong evidence of an altered landscape. Observations on mangrove forests at southeastern Brazil show that stands dominated by Laguncularia racemosa with low dbh are typical of degraded sites in process of restoration. The structure that is quite close to that of the original forest is represented by the most developed stands (high dbh and height). One of the studied stands, with mature forest of Rhizophora mangle, high developed for the structural patterns of mangrove at the southeastern Brazil is jeopardized by the invasion of Acrostichum sp., wich is preventing the development of R.mangle saplings. This invasion is related to a high deposition of mud, wich rose the substrate, reducing tide inundation.

Key words: mangrove, structure, conservation, Brazil.

\section{RESUMO}

A Lagoa da Tijuca, localizada ao sul da cidade do Rio de Janeiro, possui estreita faixa de manguezal distribuída de forma descontínua ao longo de suas margens. Os bosques de mangue apresentam grande variabilidade estrutural. Nos bosques monoespecíficos de Laguncularia racemosa, com dap médio entre 3,4 e 7,7 cm e altura média entre 4,5 e 7,7 m, há predomínio de indivíduos com menos de 10,0 $\mathrm{cm}$ de diâmetro. Nos demais pontos, observamos bosques mistos, mais desenvolvidos, dominados ora por Laguncularia racemosa, ora por Rhizophora mangle (dap médio entre 7,8 e 16,7 cm; altura média entre 6,8 e $16,1 \mathrm{~m}$ ), ora por Avicennia schaueriana (dap médio de $7,1 \mathrm{~cm}$ e altura média de 5,3 m). A variabilidade estrutural observada é forte indício de área alterada. Observações feitas em manguezais do Sudeste do Brasil demonstram que bosques dominados por Laguncularia racemosa de pequeno porte são característicos de locais alterados em processo de recomposição. Um dos pontos estudados, caracterizado por bosque maduro de Rhizophora mangle, bastante desenvolvido para os padrões estruturais do litoral sudeste do Brasil, encontra-se comprometido pela presença de Acrostichum sp., espécie invasora 
que está inibindo o desenvolvimento de plântulas de $R$. mangle. $\mathrm{O}$ fato está relacionado à alta deposição de sedimentos finos, elevando a cota do terreno e reduzindo a influência das marés.

Palavras-chave: manguezal, estrutura vegetal, conservação, Brasil.

\section{INTRODUÇÃO}

A caracterização estrutural da vegetação dos manguezais constitui valiosa ferramenta no que concerne à resposta desse ecossistema às condições ambientais existentes, bem como aos processos de alteração do meio ambiente, auxiliando, assim, nos estudos e ações que objetivam a conservação desse ecossistema.

Dessa forma, a estrutura vegetal dos bosques de mangue é uma resposta direta às condições locais. Tal fato fica evidenciado quando comparamos o desenvolvimento estrutural de espécies de mangue ao longo de gradientes latitudinal (Cintrón et al., 1985; Schaeffer-Novelli et al., 1990; Twilley, 1995), de salinidade (Cintrón et al., 1978, 1985; Cintrón \& Schaeffer-Novelli, 1982, 1983; Lugo, 1990; Lugo et al., 1990a; Cintrón-Molero, 1993; Jimenez, 1994; Twilley, 1995) e, em locais com diferentes níveis de aporte de nutrientes (Lugo \& Snedaker, 1974; Brown \& Lugo, 1982; Cintrón \& Schaeffer-Novelli, 1983; Cintrón et al., 1985; Lugo et al., 1990a; Twilley, 1995). Casos esses que são esquematicamente apresentados por diversos autores (Lugo \& Snedaker, 1974; Cintrón \& Schaeffer-Novelli, 1983,1985; Cintrón et al., 1985; Lugo et al., 1990b; Schaeffer-Novelli et al., 1990; Twilley, 1995) no modelo de "assinatura energética", no qual o manguezal possui seus processos, tais como produção primária, respiração, ciclagem de nutrientes e troca de matéria orgânica com ecossistemas adjacentes, além do desenvolvimento estrutural, controlados por forças subsidiárias.

Estas forças subsidiárias podem ser: energia solar, aporte de água doce e nutrientes e energia das marés, as quais, combinadas em diversas intensidades, vão controlar os citados atributos de cada manguezal. Segundo Cintrón-Molero \& SchaefferNovelli (1992) a capacidade dos manguezais de se manterem sob diversas condições ambientais deve-se ao seu alto grau de plasticidade fenológica, no que concerne à forma de crescimento. Assim, segundo o modelo de "assinatura energética", a estrutura dos bosques de mangue é resultado, além da combinação das diversas forças subsidiárias, do efeito da ação de tensores naturais ou antrópicos sobre o sistema (Lugo et al., 1980, 1990b; Brown \& Lugo, 1982).

Apesar da presença de grandes áreas de manguezais ao longo do litoral do Estado do Rio de Janeiro, os estudos dos atributos estruturais, funcionais e da dinâmica deste ecossistema são praticamente inexistentes no Estado, em que podemos citar o trabalho de Silva et al. (1991). Os dados existentes limitam-se a descrever a ocorrência das espécies e sua distribuição. Logo, torna-se difícil a determinação de um padrão estrutural para os manguezais do Estado do Rio de Janeiro, principalmente se considerarmos a plasticidade estrutural anteriormente descrita.

Schaeffer-Novelli et al. (1990), com base no relevo, tipo de solo, cobertura vegetal, temperatura média anual, evapotranspiração potencial, amplitude das marés médias e de sizígia, dividiram o litoral brasileiro em oito unidades fisiográficas, estando os manguezais do Rio de Janeiro na unidade VII, os quais, por estarem submetidos a condições ambientais regionais similares, teriam um desenvolvimento estrutural dentro de uma mesma faixa. No entanto, não podemos negligenciar as características ambientais de caráter local (Smith III, 1992; Ogino, 1993), as quais determinam uma variabilidade estrutural e funcional dentro de cada unidade proposta por Schaeffer-Novelli et al. (1990), além dos tensores naturais e de origem antrópica.

Assim, com o objetivo de determinar a situação dos manguezais da Lagoa da Tijuca, bem como levantar a característica estrutural mais próxima dos bosques originais da região, realizouse o presente estudo, que constitui parte de relatório apresentado pelo Departamento de Oceanografia da Universidade do Estado do Rio de Janeiro, sobre a faixa marginal da referida lagoa (Zee, 1993).

\section{Características da Baixada de Jacarepaguá}

A Baixada de Jacarepaguá, localizada ao sul do município do Rio de Janeiro, está compreendida entre as latitudes $22^{\circ} 55^{\prime} \mathrm{S}$ e $23^{\circ} 03^{\prime} \mathrm{S}$ e as longitudes 
$43^{\circ} 30^{\prime} \mathrm{W}$ e $43^{\circ} 18^{\prime} \mathrm{W}$. É delimitada pelos maciços montanhosos da Tijuca à leste, da Pedra Branca à oeste e pelo Oceano Atlântico ao sul (Fig. 1).

Sua área total, com aproximadamente 130 $\mathrm{km}^{2}$, é ocupada em parte por um sistema de lagoas, segundo descrição de Strang et al. (1965): Jacarepaguá-Camorim-Tijuca, $8,8 \mathrm{~km}^{2}$, Marapendi, 4,6 $\mathrm{km}^{2}$, e Lagoinha com $0,2 \mathrm{~km}^{2}$. No entanto, dados mais recentes apontam uma redução na área desse complexo lagunar, possuindo as lagoas de Marapendi e Lagoinha $3,32 \mathrm{~km}^{2}$, Lagoa da Tijuca 3,25 $\mathrm{km}^{2}$, Camorim $1,03 \mathrm{~km}^{2}$ e a Lagoa de Jacarepaguá $3,25 \mathrm{~km}^{2}$ (CEDAE, 1987).

O complexo lagunar da Baixada de Jacarepaguá é caracterizado por lagoas de pequena profundidade, sendo, segundo Marques (1990), composto por $60 \%$ de área com profundidades inferiores a $1 \mathrm{~m}$ e $10 \%$ com profundidades entre 5 e 12 m. Zee (1992), utilizando dados de 1977, determinou uma profundidade média de 2,09 $\mathrm{m}$ para a Lagoa da Tijuca. Profundidade esta que, devido ao acelerado processo de assoreamento do citado complexo lagunar (Marques, 1990; Zee, 1992), é, certamente, bem menor atualmente.

O clima da região, utilizando-se dados da estação meteorológica do Aeroporto de Jacarepaguá, pode ser classificado, segundo Köppen, como do tipo Am. A precipitação média anual é de $1116,4 \mathrm{~mm}$ e a temperatura média anual do ar é de $24,6^{\circ} \mathrm{C}$. Podemos observar, ainda, que o clima é predominantemente úmido, com pequeno período seco no mês de agosto.

Como podemos observar na Fig. 1, esse complexo lagunar encontra-se interligado, sendo a Lagoa de Camorim o ponto de ligação entre as lagoas de Jacarepaguá e Tijuca e o Canal de Marapendi a ligação das lagoas de Marapendi e Tijuca. Estando esta, por sua vez, em contato direto e permanente com o mar, através do Canal da Barra da Tijuca. Dessa forma, é a lagoa da Tijuca aquela que sofre maior influência das águas marinhas.

Zee (1993) relata algumas características fisico-químicas para as águas da Lagoa da Tijuca, na área estudada. Assim, observou-se uma variação do $\mathrm{pH}$, com as marés, entre 7,0 e 9,4 .

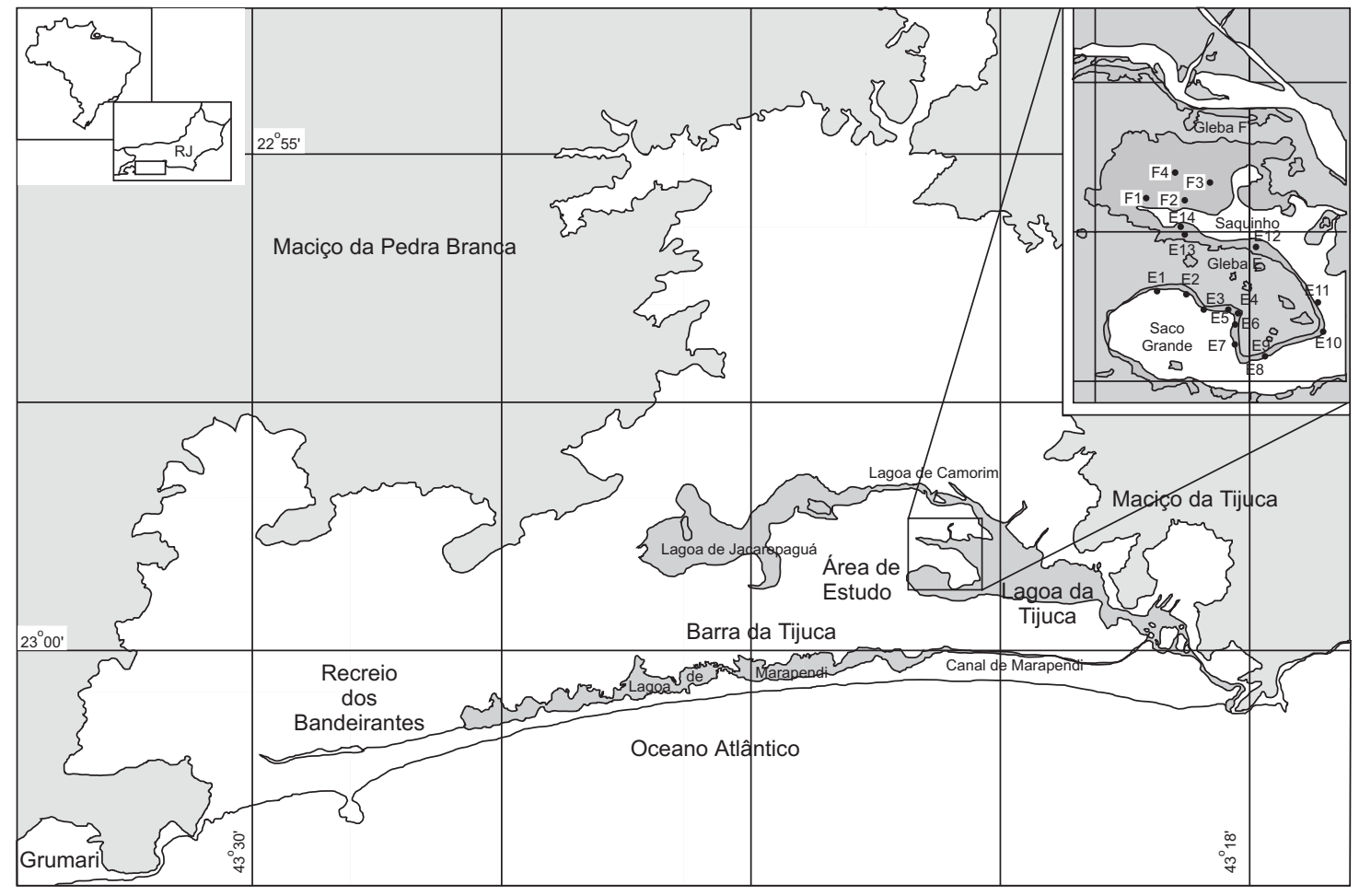

Fig. 1 - Região da Baixada de Jacarepaguá, com detalhe da área de estudo na Lagoa da Tijuca. 
Para a salinidade, Zee (1993) observou um comportamento associado às marés de quadratura e de sizígia e variações com a profundidade, obtendo valores entre 5,5 e 28,8. Esse autor descreve ainda uma penetração de água do mar, pelo fundo, na região do Saco Grande, a qual possui maior salinidade. Foi observada, ainda, supersaturação de oxigênio dissolvido na área do Saco Grande, em oposição às demais áreas do fundo da lagoa.

A ocorrência de manguezais é marcada ao longo desse sistema lagunar, sendo mais significativa na Lagoa da Tijuca, na qual há maior influência das marés, sendo observados nesse local os bosques mais desenvolvidos, apresentando-se, no entanto, distribuídos de forma descontínua ao longo de suas margens, devido a diversos processos de origem antrópica que vêm se manifestando ao longo dos anos.

\section{MATERIAL E MÉTODOS}

$\mathrm{O}$ estudo abrangeu as áreas conhecidas como Saco Grande e Saquinho (Fig. 1), pois é nessa região que se encontram os principais remanescentes de manguezal do sistema lagunar da Baixada de Jacarepaguá.

A metodologia adotada seguiu, em linhas gerais, a metodologia proposta por Cintrón \& Schaeffer-Novelli (1984) e Schaeffer-Novelli \& Cintrón (1986).

As 18 estações de coleta foram fixadas segundo a heterogeneidade das características estruturais gerais da vegetação. Assim, foram selecionadas as áreas de amostragem, segundo o mosaico estrutural presente na região, após análise de fotos aéreas, e em cada uma das áreas selecionadas foram delimitadas parcelas, de forma aleatória, buscandose retratar o aspecto geral da estação escolhida. As parcelas utilizadas possuíam $10 \mathrm{~m}$ x $10 \mathrm{~m}$, exceto na estação E9 (10 m x $5 \mathrm{~m})$ e na estação F4 $(10 \mathrm{~m} \times 20 \mathrm{~m})$. No primeiro caso, tais medidas foram adotadas devido à estreita faixa de mangue associada à alta densidade de troncos e, no segundo caso, devido ao alto desenvolvimento estrutural do bosque (baixa densidade com indivíduos bem desenvolvidos).

Apesar da não realização de réplicas, o tamanho das parcelas foi definido de forma a garantir a representatividade amostral do ponto de coleta, segundo o descrito por Cintrón \& SchaefferNovelli (1984) e Schaeffer-Novelli \& Cintrón
(1986). Todos os indivíduos com altura superior a $1 \mathrm{~m}$, de cada parcela, foram identificados e tiveram medidos o diâmetro à altura do peito (dap) e a altura. No caso dos indivíduos menores, o dap foi substituído pelo diâmetro do tronco abaixo da primeira ramificação, o qual, para efeito prático, será, doravante, também referido como dap. O dap foi medido com trena e a altura foi medida utilizando-se telêmetro ótico (ou trena para os indivíduos menores).

Posteriormente, foram calculadas as densidades de troncos e indivíduos vivos e mortos de cada espécie, para cada parcela, a área basal viva e morta, o dap médio e a altura média do bosque em cada ponto de coleta e as dominâncias em área basal e de troncos vivos e mortos. Em seguida, foi realizada análise de agrupamento (UPGMA) das estações de coleta, através da análise dos atributos estruturais: densidade relativa de troncos de cada espécie, altura, dap, percentual de área basal viva e contribuição (\%) de área basal de indivíduos maiores que $10 \mathrm{~cm}$ de dap. Para tanto, foi utilizado o programa estatístico "Statistica".

Por fim, foi feita a análise comparativa de fotos aéreas dos anos de 1975 e 1993, além de medições da largura da faixa de manguezal nas estações de amostragem para detectarmos alterações na área ocupada por esse ecossistema na região de estudo.

\section{RESULTADOS}

Ao analisarmos os dados de altura e dap das estações de coleta (Tabela 1), notamos grande heterogeneidade na estrutura vegetal entre os pontos de amostragem. Nessa tabela, podemos observar, ainda, grande diferença entre indivíduos altos (basicamente Rhizophora mangle) e indivíduos baixos, caracterizando um mosaico estrutural, tanto em termos de dap, como em termos de altura. Esta heterogeneidade também é notada na ocorrência das três espécies de mangue em cada estação.

Em relação à área basal (Tabela 2), também é notada uma grande variabilidade, principalmente no que diz respeito à área basal viva, fato que não é tão evidente para as estações E7, E10, E11, E13 e F14, com domínio de $R$. mangle, nas quais a área basal não foi muito variável. Já em relação à área basal morta, observamos menor variabilidade, estando os valores entre 0 e $5,0 \mathrm{~m}^{2} / \mathrm{ha}$, excessão feita à estação $\mathrm{F} 3$, que apresentou valor muito elevado. 
A proporção de área basal viva em relação à área basal morta (Tabela 3) foi bastante elevada em todas as estações, excetuando-se a estação F3. Nas estações E1 e E2, encontramos espécies vegetais que não são típicas de manguezal, sendo a contribuição destas bem modesta em área basal (Tabela 3). De forma geral, observamos uma maior contribuição de área basal viva de indivíduos com dap acima de 2,5 cm (Tabela 2), sendo que nas estações E5, E6, E12, E14, F1, F2 e F3 esse domínio se faz na classe intermediária (dap entre 2,5 e 10,0 cm). Todas essas estações, com exceção da estação E5, constituem bosques monoespecíficos de Laguncularia racemosa.

A distribuição dos troncos em classes de dap (Tabela 4), nos bosques monoespecíficos de $L$. racemosa, também demonstra predomínio de troncos de diâmetro entre 2,5 e $10,0 \mathrm{~cm}$, cabendo ressaltar a maior ocorrência de troncos acima de 10,0 $\mathrm{cm}$ de dap nas estações F1 e F2, quando comparadas às demais, e alta taxa de indivíduos mor- tos nas estações E9 e F3. Através da análise da Tabela 3, observamos um grande domínio, em área basal, de L.racemosa na maior parte das estações estudadas.

Ainda em relação à distribuição dos troncos em classes de dap (Tabela 4), notamos predomínio de troncos de diâmetro reduzido (dap $<2,5 \mathrm{~cm}$ ) nas estações E1, E2 e E11 e de troncos intermediários (dap entre 2,5 cm e 10,0 cm) nas estações E3, E4, E5, E7 e E13. No entanto, a maior contribuição em área basal, nas estações E1, E2, E3, E7, E11 e E13, se dá para indivíduos acima de 10,0 $\mathrm{cm}$ de dap, ao contrário das estações E4 e E5, em que a área basal de indivíduos entre $2,5 \mathrm{~cm}$ e 10,0 $\mathrm{cm}$ de dap é mais representativa (Tabela 2). Por fim, cabe destacar a alta contribuição de troncos acima de 10,0 cm de dap nas estações E13 e F4. Outro ponto importante a ressaltar é a não ocorrência de indivíduos mortos com mais de 10,0 cm de dap (Tabela 4). Fato este observado em todas as estações de amostragem, exceto nas estações E8 e F4.

TABELA 1

Número de troncos vivos amostrados (N), altura média e dap médio dos bosques de mangue da Lagoa da Tijuca.

\begin{tabular}{|c|c|c|c|c|c|}
\hline \multirow{2}{*}{ Estação } & \multirow{2}{*}{$\mathbf{N}$} & \multicolumn{3}{|c|}{ Altura média do bosque (m) } & \multirow{2}{*}{$\begin{array}{c}\text { dap } \\
\text { (cm) }\end{array}$} \\
\hline & & Inferior & Superior & Total & \\
\hline E1 & 77 & 3,46 & 7,20 & 4,12 & 5,81 \\
\hline E2 & 113 & 2,29 & 7,71 & 3,55 & 6,83 \\
\hline E3 & 34 & 3,89 & 8,80 & 5,81 & 11,07 \\
\hline E4 & 63 & 3,54 & 6,62 & 6,32 & 5,47 \\
\hline E5 & 93 & 4,20 & 6,55 & 6,24 & 4,80 \\
\hline E6 & 109 & 3,24 & 6,56 & 5,99 & 5,00 \\
\hline E7 & 42 & 2,65 & 6,79 & 4,60 & 8,44 \\
\hline E8 & 49 & 2,51 & 7,56 & 5,34 & 7,05 \\
\hline E9 & 51 & 4,49 & 7,28 & 6,86 & 7,18 \\
\hline E10 & 41 & 3,11 & 9,66 & 4,28 & 8,37 \\
\hline E11 & 44 & 1,49 & 10,46 & 3,37 & 7,79 \\
\hline E12 & 170 & 3,72 & 5,43 & 4,52 & 3,40 \\
\hline E13 & 28 & 2,94 & 7,32 & 5,92 & 8,69 \\
\hline E14 & 73 & 3,41 & 7,18 & 6,18 & 5,83 \\
\hline F1 & 48 & 3,35 & 8,06 & 7,66 & 7,66 \\
\hline $\mathrm{F} 2$ & 32 & 4,03 & 7,79 & 7,39 & 7,54 \\
\hline F3 & 70 & 3,37 & 7,97 & 7,51 & 5,10 \\
\hline $\mathrm{F} 4$ & 25 & 1,90 & 16,13 & 11,58 & 16,67 \\
\hline
\end{tabular}


TABELA 2

Área basal (m²/ha) viva e morta, por classe de dap, dos bosques de mangue da Lagoa da Tijuca.

\begin{tabular}{|c|c|c|c|c|c|c|c|c|c|}
\hline \multirow[b]{2}{*}{ Estações } & \multicolumn{4}{|c|}{ Área basal viva $\left(\mathrm{m}^{2} / \mathrm{ha}\right)$} & \multicolumn{4}{|c|}{ Área basal morta $\left(\mathrm{m}^{2} / \mathrm{ha}\right)$} & \multirow{2}{*}{$\begin{array}{l}\text { Total } \\
\text { geral }\end{array}$} \\
\hline & $\begin{array}{l}<2,5 \\
(\mathrm{~cm})\end{array}$ & $\begin{array}{l}>2,5 \\
(\mathrm{~cm})\end{array}$ & $\begin{array}{c}>10,0 \\
(\mathrm{~cm})\end{array}$ & Total & $\begin{array}{l}<2,5 \\
(\mathrm{~cm})\end{array}$ & $\begin{array}{l}>2,5 \\
(\mathrm{~cm})\end{array}$ & $\begin{array}{c}>10,0 \\
(\mathrm{~cm})\end{array}$ & Total & \\
\hline E1 & 0,69 & 7,83 & 11,89 & 20,41 & 0,21 & 0,42 & - & 0,63 & 21,04 \\
\hline E2 & 1,51 & 4,17 & 35,67 & 41,35 & 0,21 & 0,58 & - & 0,79 & 42,14 \\
\hline E3 & 0,12 & 2,83 & 29,78 & 32,73 & - & - & - & - & 32,73 \\
\hline $\mathrm{E} 4$ & 0,25 & 8,11 & 6,45 & 14,81 & 0,02 & 0,07 & - & 0,09 & 14,90 \\
\hline E5 & 0,08 & 15,29 & 1,47 & 16,84 & 0,05 & 0,81 & - & 0,86 & 17,70 \\
\hline E6 & 0,38 & 17,49 & 3,49 & 21,36 & 0,66 & 1,70 & - & 2,36 & 23,72 \\
\hline E7 & 0,17 & 7,09 & 16,25 & 23,51 & 0,03 & 0,15 & - & 0,18 & 23,69 \\
\hline E8 & 0,43 & 3,94 & 14,77 & 19,14 & - & 1,13 & 2,46 & 3,59 & 22,73 \\
\hline E9 & 0,42 & 13,24 & 27,71 & 41,37 & 2,43 & 2,12 & - & 4,55 & 45,92 \\
\hline E10 & 0,41 & 3,00 & 19,19 & 22,54 & 0,03 & 0,35 & - & 0,38 & 22,92 \\
\hline E11 & 0,38 & 0,38 & 20,20 & 20,96 & - & - & - & - & 20,96 \\
\hline E12 & 1,49 & 13,95 & - & 15,44 & 0,57 & 1,42 & - & 1,99 & 17,43 \\
\hline E13 & 0,06 & 3,29 & 13,27 & 16,62 & - & - & - & - & 16,62 \\
\hline E14 & 0,04 & 17,57 & 1,88 & 19,49 & 1,18 & 3,47 & - & 4,65 & 24,14 \\
\hline $\mathrm{F} 1$ & 0,02 & 15,01 & 7,09 & 22,12 & - & 1,92 & - & 1,92 & 24,04 \\
\hline $\mathrm{F} 2$ & - & 9,36 & 4,92 & 14,28 & - & 0,97 & - & 0,97 & 15,25 \\
\hline F3 & - & 14,25 & - & 14,25 & 1,40 & 13,79 & - & 15,19 & 29,44 \\
\hline F4 & 0,08 & - & 27,19 & 27,27 & - & 0,08 & 1,03 & 1,11 & 28,38 \\
\hline
\end{tabular}

Através da análise de agrupamentos, baseada na densidade relativa de troncos vivos das três espécies de mangue (Fig. 2a), podemos identificar a existência de agrupamentos das estações, segundo o domínio das espécies vegetais.

No que diz respeito ao desenvolvimento estrutural dos bosques estudados, observamos na Fig. $2 \mathrm{~b}$, através da análise de agrupamentos baseada em parâmetros estruturais, dois grandes grupos quanto ao desenvolvimento estrutural. O primeiro grupo, composto pelas estações E4, E5, E6, E12, E14, F1, F2 e F3, constitui-se de estações com contribuição, em área basal, de indivíduos acima de $10,0 \mathrm{~cm}$ de dap, inferior a $50 \%$ (Tabela 2). Nesse grupo, identificamos ainda três subgrupos. O primeiro é composto pelas estações E4, F1 e F2, com maior desenvolvimento estrutural. O segundo subgrupo (estações E5, E6, E12 e E14) é menos desenvolvido, com altura e dap inferiores ao primeiro subgrupo. Por fim, encontramos a estação F3, que, ao contrário das demais estações do agrupamento, que possuem de $80 \%$ a $100 \%$ de área basal viva, possui menos de 50\% de área basal viva (Tabela 2 ).

O outro agrupamento, quanto ao desenvolvimento estrutural, é composto pelas estações E1, E2, E3, E7, E8, E9, E10, E11, E13 e F4, caracterizadas por possuírem contribuição de área basal de indivíduos acima de 10,0 $\mathrm{cm}$ de dap, superior a 50\% (Tabela 2). Neste grupo identificamos dois subgrupos. O primeiro subgrupo é composto pelas estações E2, E3, E10, E11, E13 e F4; neste observamos estações com mais de $85 \%$ de área basal relacionada a indivíduos com dap acima de 10,0 $\mathrm{cm}$, exceto para a estação E13, na qual essa contribuição foi de aproximadamente $80 \%$. Esse subgrupo ainda é caracterizado por possuir as maiores alturas máximas (Tabela 1) do agrupamento, além dos maiores dap médios de todas as estações estudadas. No caso da estação F4 (Fig. 2b), a mesma destaca-se dentro do subgrupo por possuir o maior desenvolvimento estrutural de todas as estações estudadas (maiores dap, altura e contribuição de área basal de indivíduos acima de 10,0 cm de dap). 
TABELA 3

Número de troncos por hectare (n) e dominância em área basal (\%)

viva e morta, por espécie, nos bosques de mangue da Lagoa da Tijuca.

\begin{tabular}{|c|c|c|c|c|c|c|c|c|}
\hline \multirow{2}{*}{ Estação } & \multicolumn{4}{|c|}{ Área basal viva (\%) } & \multicolumn{4}{|c|}{ Área basal morta (\%) } \\
\hline & $\mathbf{R h}(\mathbf{n})$ & $\operatorname{Lg}(\mathrm{n})$ & Av (n) & $\left(\mathrm{m}^{2} / \mathrm{ha}\right)$ & Rh (n) & $\operatorname{Lg}(\mathbf{n})$ & $\operatorname{Av}(n)$ & $\left(\mathrm{m}^{2} / \mathrm{ha}\right)$ \\
\hline E1 & $\begin{array}{c}10,60 \\
(1100)\end{array}$ & $\begin{array}{c}73,86 \\
(5900)\end{array}$ & $\begin{array}{l}12,50 \\
(700)\end{array}$ & 20,41 & - & $\begin{array}{c}2,95 \\
(1400)\end{array}$ & - & 0,63 \\
\hline $\mathrm{E} 2 *$ & $\begin{array}{l}23,49 \\
(400)\end{array}$ & $\begin{array}{l}24,23 \\
(8200)\end{array}$ & $\begin{array}{l}46,94 \\
(600)\end{array}$ & 41,35 & - & $\begin{array}{c}1,76 \\
(1500)\end{array}$ & - & 0,79 \\
\hline E3 & $\begin{array}{l}16,99 \\
(400)\end{array}$ & $\begin{array}{l}66,70 \\
(2400)\end{array}$ & $\begin{array}{l}16,25 \\
(600)\end{array}$ & 32,73 & - & - & - & - \\
\hline E4 & $\begin{array}{l}36,11 \\
(400)\end{array}$ & $\begin{array}{c}54,90 \\
(5000)\end{array}$ & $\begin{array}{c}8,19 \\
(900)\end{array}$ & 14,81 & - & $\begin{array}{c}0,60 \\
(200)\end{array}$ & - & 0,09 \\
\hline E5 & $\begin{array}{c}8,31 \\
(103)\end{array}$ & $\begin{array}{l}81,98 \\
(8796)\end{array}$ & $\begin{array}{r}4,80 \\
(402)\end{array}$ & 16,84 & - & $\begin{array}{l}4,80 \\
(999)\end{array}$ & - & 0,86 \\
\hline E6 & - & $\begin{array}{c}90,05 \\
(10900)\end{array}$ & - & 21,36 & - & $\begin{array}{c}9,95 \\
(4800)\end{array}$ & - & 2,36 \\
\hline E7 & $\begin{array}{c}46,01 \\
(1300)\end{array}$ & $\begin{array}{l}29,97 \\
(1800)\end{array}$ & $\begin{array}{c}23,17 \\
(1100)\end{array}$ & 23,51 & $\begin{array}{c}0,38 \\
(100)\end{array}$ & $\begin{array}{c}0,08 \\
(200)\end{array}$ & $\begin{array}{c}0,21 \\
(100)\end{array}$ & 0,18 \\
\hline E8 & $\begin{array}{c}5,63 \\
(400)\end{array}$ & $\begin{array}{l}21,87 \\
(1400)\end{array}$ & $\begin{array}{c}56,67 \\
(3100)\end{array}$ & 19,14 & - & $\begin{array}{l}13,46 \\
(200)\end{array}$ & $\begin{array}{l}2,29 \\
(200)\end{array}$ & 3,59 \\
\hline E9 & - & $\begin{array}{c}90,09 \\
(10200)\end{array}$ & - & 41,37 & - & $\begin{array}{c}9,89 \\
(15400)\end{array}$ & - & 4,55 \\
\hline E10 & $\begin{array}{l}83,42 \\
(498)\end{array}$ & $\begin{array}{c}14,88 \\
(3600)\end{array}$ & - & 22,54 & - & $\begin{array}{l}1,66 \\
(602)\end{array}$ & - & 0,38 \\
\hline E11 & $\begin{array}{c}99,76 \\
(4000)\end{array}$ & $\begin{array}{c}0,24 \\
(400)\end{array}$ & - & 20,96 & - & - & - & - \\
\hline E12 & - & $\begin{array}{c}88,58 \\
(17002)\end{array}$ & - & 15,44 & - & $\begin{array}{c}11,42 \\
(4198)\end{array}$ & - & 1,99 \\
\hline E13 & $\begin{array}{c}99,58 \\
(2500)\end{array}$ & $\begin{array}{c}0,36 \\
(300)\end{array}$ & - & 16,62 & - & - & - & - \\
\hline E14 & - & $\begin{array}{c}80,74 \\
(7306)\end{array}$ & - & 19,49 & - & $\begin{array}{l}19,22 \\
(2294)\end{array}$ & - & 4,65 \\
\hline $\mathrm{F} 1$ & - & $\begin{array}{c}91,97 \\
(4800)\end{array}$ & - & 22,12 & - & $\begin{array}{c}7,99 \\
(1000)\end{array}$ & - & 1,92 \\
\hline F2 & - & $\begin{array}{c}93,57 \\
(3200) \\
\end{array}$ & - & 14,28 & - & $\begin{array}{r}6,36 \\
(500) \\
\end{array}$ & - & 0,97 \\
\hline F3 & - & $\begin{array}{c}48,40 \\
(7000) \\
\end{array}$ & - & 14,25 & - & $\begin{array}{c}51,56 \\
(18500) \\
\end{array}$ & - & 15,19 \\
\hline $\mathrm{F} 4$ & $\begin{array}{c}96,02 \\
(1200) \\
\end{array}$ & $\begin{array}{l}0,04 \\
(50) \\
\end{array}$ & - & 27,27 & - & $\begin{array}{c}3,91 \\
(100) \\
\end{array}$ & - & 1,11 \\
\hline
\end{tabular}

* Inclui ainda $3,46 \%$ de área basal viva e $0,12 \%$ de área basal morta, correspondente a 2.200 troncos/ha de espécies que não são típicas de manguezal.

$\mathrm{Rh}=$ Rhizophora mangle $\mathrm{Lg}=$ Laguncularia racemosa $; \mathrm{Av}=$ Avicennia schaueriana . Valores entre parênteses correspondem ao número de troncos estimados por hectare. 
TABELA 4

Densidade relativa (\%) de troncos vivos e mortos, por espécie e por classe de dap, nos bosques de mangue da Lagoa da Tijuca.

\begin{tabular}{|c|c|c|c|c|c|c|c|c|c|c|}
\hline \multirow{3}{*}{ Estação } & & & & \multicolumn{3}{|c|}{ Troncos vivos } & & & & \multirow{3}{*}{$\begin{array}{c}\text { Total } \\
\text { (troncos/ha) }\end{array}$} \\
\hline & \multicolumn{3}{|c|}{$<2,5 \mathrm{~cm}$} & \multicolumn{3}{|c|}{$>2,5 \mathrm{~cm}$} & \multicolumn{3}{|c|}{$>10,0 \mathrm{~cm}$} & \\
\hline & $\mathbf{R h}$ & $\mathbf{L g}$ & Av & $\mathbf{R h}$ & Lg & Av & $\mathbf{R h}$ & $\mathbf{L g}$ & Av & \\
\hline E1 & 8,8 & 28,6 & 2,2 & 2,2 & 29,7 & 4,4 & 1,1 & 6,6 & 1,1 & 7.700 \\
\hline $\mathrm{E} 2$ & - & 39,5 & 2,3 & - & 23,3 & - & 3,1 & 0,8 & 2,3 & 11.300 \\
\hline E3 & 2,9 & 8,8 & 2,9 & 2,9 & 52,9 & 8,8 & 5,9 & 8,8 & 5,9 & 3.400 \\
\hline E4 & - & 7,7 & 1,5 & - & 67,7 & 12,3 & 6,2 & 1,5 & - & 6.300 \\
\hline E5 & - & 2,9 & - & - & 82,5 & 3,9 & 1,0 & - & - & 9.300 \\
\hline E6 & - & 11,5 & - & - & 56,1 & - & - & 1,9 & - & 10.900 \\
\hline E7 & 13,0 & 6,5 & 2,2 & 10,9 & 26,1 & 17,4 & 4,3 & 6,5 & 4,3 & 4.200 \\
\hline E8 & 3,8 & 15,1 & 17,0 & 3,8 & 5,7 & 28,3 & - & 5,7 & 13,2 & 4.900 \\
\hline E9 & - & 4,7 & - & - & 34,4 & - & - & 0,8 & - & 10.200 \\
\hline E10 & - & 36,2 & - & - & 40,4 & - & 10,6 & - & - & 4.100 \\
\hline E11 & 70,5 & 9,1 & - & 2,3 & - & - & 18,2 & - & - & 4.400 \\
\hline E12 & - & 32,1 & - & - & 48,1 & - & - & - & - & 17.000 \\
\hline E13 & 7,1 & 7,1 & - & 42,9 & 3,6 & - & 39,3 & - & - & 2.800 \\
\hline E14 & - & 2,1 & - & - & 74,0 & - & - & - & - & 7.300 \\
\hline $\mathrm{F} 1$ & - & 3,4 & - & - & 67,2 & - & - & 12,1 & - & 4.800 \\
\hline $\mathrm{F} 2$ & - & - & - & - & 73,0 & - & - & 13,5 & - & 3.200 \\
\hline F3 & - & - & - & - & 27,5 & - & - & - & - & 7.000 \\
\hline \multirow[t]{2}{*}{$\mathrm{F} 4$} & 25,9 & 3,7 & - & - & - & - & 63,0 & - & - & 1.250 \\
\hline & & & & \multicolumn{3}{|c|}{ Troncos mortos } & & & & \\
\hline \multirow{2}{*}{ Estação } & \multicolumn{3}{|c|}{$<2,5 \mathrm{~cm}$} & \multicolumn{3}{|c|}{$>2,5 \mathrm{~cm}$} & \multicolumn{3}{|c|}{$>10,0 \mathrm{~cm}$} & \multirow{2}{*}{$\begin{array}{c}\text { Total } \\
\text { (troncos/ha) }\end{array}$} \\
\hline & $\mathbf{R h}$ & Lg & Av & $\mathbf{R h}$ & $\mathbf{L g}$ & Av & $\mathbf{R h}$ & Lg & Av & \\
\hline E1 & - & 12,1 & - & - & 3,3 & - & - & - & - & 1.400 \\
\hline E2 & - & 10,1 & - & - & 1,6 & - & - & - & - & 1.600 \\
\hline E3 & - & - & - & - & - & - & - & - & - & - \\
\hline $\mathrm{E} 4$ & - & 1,5 & - & - & 1,5 & - & - & - & - & 200 \\
\hline E5 & - & 1,9 & - & - & 7,8 & - & - & - & - & 1.000 \\
\hline E6 & - & 21,7 & - & - & 8,9 & - & - & - & - & 4.800 \\
\hline E7 & - & 4,3 & - & 2,2 & - & 2,2 & - & - & - & 400 \\
\hline E8 & - & - & - & - & 1,9 & 3,8 & - & 1,9 & - & 400 \\
\hline E9 & - & 46,1 & - & - & 14,1 & - & - & - & - & 15.400 \\
\hline E10 & - & 6.4 & - & - & 6,4 & - & - & - & - & 600 \\
\hline E11 & - & - & - & - & - & - & - & - & - & - \\
\hline E12 & - & 12,3 & - & - & 7,5 & - & - & - & - & 4.200 \\
\hline E13 & - & - & - & - & - & - & - & - & - & - \\
\hline E14 & - & 3.1 & - & - & 20.8 & - & - & - & - & 2.300 \\
\hline $\mathrm{F} 1$ & - & - & - & - & 17,2 & - & - & - & - & 1.000 \\
\hline $\mathrm{F} 2$ & - & - & - & - & 13,5 & - & - & - & - & 500 \\
\hline $\mathrm{F} 3$ & - & 14,9 & - & - & 57.6 & - & - & $\ldots$ & - & 18.500 \\
\hline $\mathrm{F} 4$ & - & $\ldots$ & - & - & 3.7 & - & - & 3.7 & - & 100 \\
\hline
\end{tabular}

$\mathrm{Rh}=$ Rhizophora mangle $; \mathrm{Lg}=$ Laguncularia racemosa $; \mathrm{Av}=$ Avicennia schaueriana . 


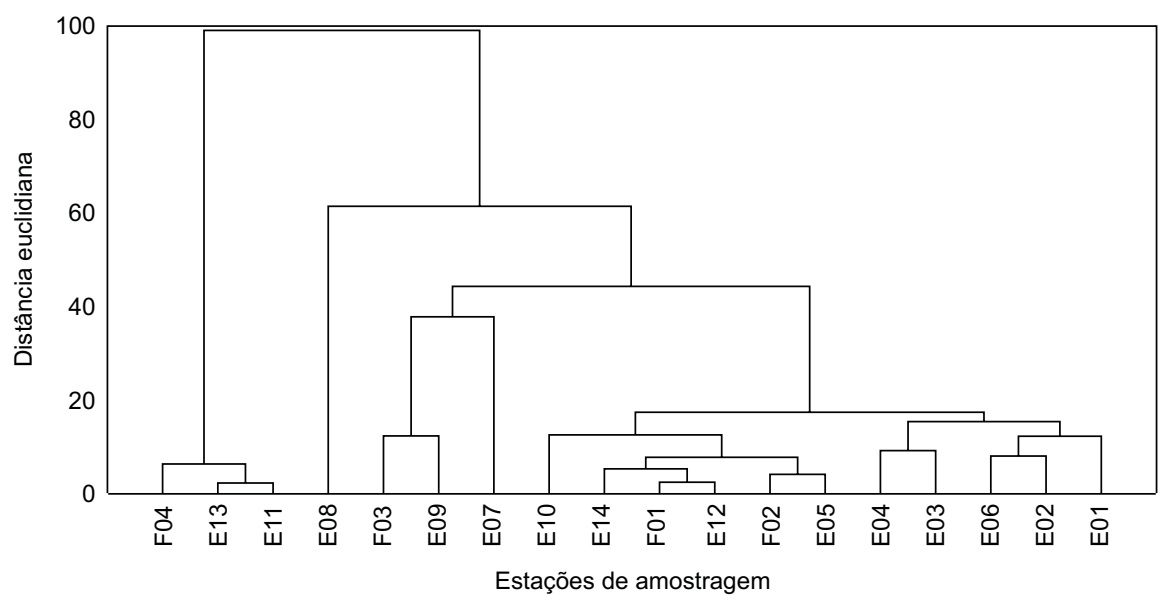

Fig. 2a - Análise de agrupamento (UPGMA) das estações de amostragem, segundo a densidade relativa das espécies vegetais típicas de manguezal.

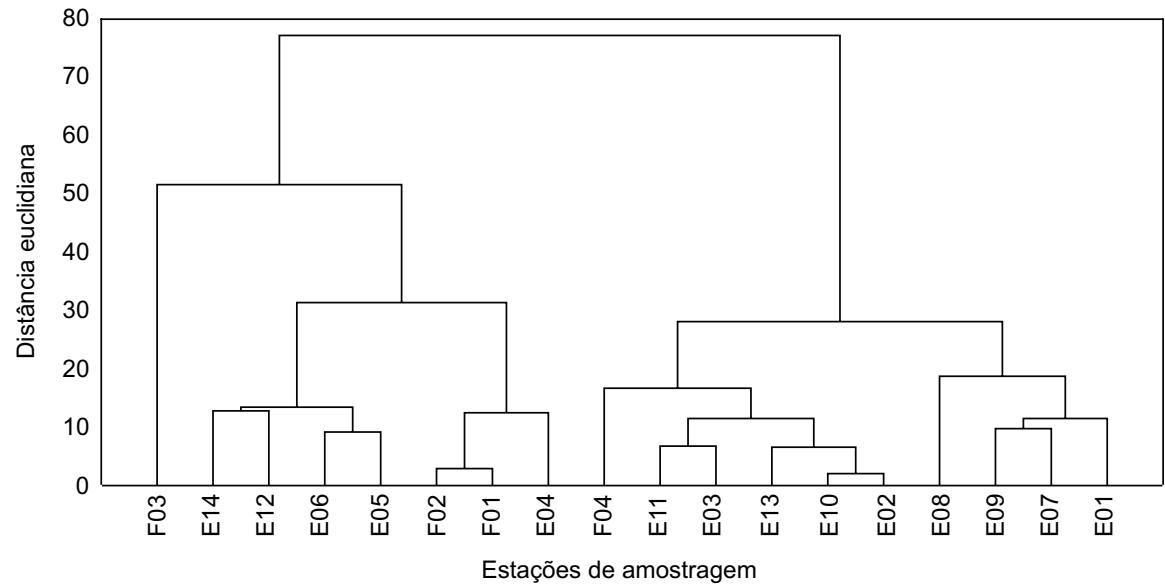

Fig. 2b - Análise de agrupamento (UPGMA) das estações de amostragem, segundo o desenvolvimento estrutural (altura, dap, percentual de área basal viva e percentual de área basal de indivíduos maiores que $10,0 \mathrm{~cm}$ de dap).

O outro subgrupo desse agrupamento é composto pelas estações E1, E7, E8 e E9, as quais possuem contribuição entre $50 \%$ e $78 \%$ de área basal de indivíduos acima de $10,0 \mathrm{~cm}$ de dap. A diferenciação da estação E8 nesse subgrupo está relacionada ao fato de essa ser a única estação com menos de $90 \%$ de área basal viva do subgrupo em questão (Tabela 2).

Assim, os dendogramas baseados na densidade relativa e no desenvolvimento estrutural (Figs. $2 \mathrm{a}$ e $2 \mathrm{~b}$ ) demonstraram padrões de agrupamentos distintos, com o segundo (Fig. 2b) separando melhor as estações estudadas. Dessa forma, a den- sidade não seria a melhor variável a ser utilizada na comparação dos pontos estudados.

\section{DISCUSSÃO}

Da análise dos dados estruturais, verificase claramente grande variabilidade no desenvolvimento estrutural entre os diversos pontos de amostragem, destacando-se um maior desenvolvimento em algumas estações, caracterizando um mosaico estrutural.

A descontinuidade da estrutura dos bosques de mangue na área de estudo está de acordo com 
observações de Araújo (1978), que faz referência a um manguezal bem desenvolvido, porém nãocontínuo, na Lagoa da Tijuca, sendo grande a ocorrência de Hibiscus sp. e Acrostichum sp. devido à destruição dos manguezais.

Há outros aspectos que nos levam a concluir que os manguezais dessa região se encontram sob influência de algum tipo de tensor. O principal indício é a ocorrência de manchas estruturalmente bem desenvolvidas, com alturas médias e dap superiores aos de outras áreas dentro da própria "Gleba E". Podemos citar ainda a ocorrência isolada, ao longo de toda a área, de indivíduos de grande porte das três espécies. Tais características vão ao encontro da teoria de mosaico de manchas com diferentes níveis de desenvolvimento estrutural em ecossistemas florestais (Smith III, 1992).

Essa heterogeneidade estrutural é forte indicador de área alterada. Peria et al. (1990) observaram, para bosques em recomposição, valores reduzidos de diâmetro e altura médios como resultado de intervenções humanas recorrentes. Tal fato também é citado por Jimenez et al. (1985), que afirmam que o desenvolvimento estrutural reduzido pode ser devido a alterações antrópicas, as quais não permitem que o manguezal atinja sua maturidade estrutural. Lugo \& Zucca (1977) colocam a alta densidade de árvores como conseqüência de "destruições" periódicas causadas pelas geadas.

A diversidade estrutural encontrada na região de estudo pode ser um exemplo do referido por Lugo \& Snedaker (1973), em que afirmam que distúrbios em florestas "estáveis" de mangue permitem a colonização por diversas espécies de mangue (maior diversidade) e, com o tempo, esta diversidade se reduz devido à seleção do ambiente, retornando a um bosque monoespecífico. Da mesma forma, Smith III (1992) relata que manguezais sujeitos a maior freqüência de distúrbios possuem maior número de espécies vegetais. Podemos dizer, assim, que os bosques da "Gleba E" foram de alguma forma alterados, apresentando estrutura pouco desenvolvida, e que a estrutura mais semelhante à original estaria representada pelas estações estruturalmente mais desenvolvidas. Tais bosques podem ser encontrados no agrupamento das estações E2, E3, E10, E11, E13 e F4, observado na Fig. 2b, na qual encontramos bosques com contribuição em área basal de indivíduos com dap acima de $10,0 \mathrm{~cm}$ superior a $80 \%$, os maiores dap médios de toda a área estudada; grande desenvolvimento em termos de altura; e alta taxa de área basal viva - características essas que, juntamente com baixa densidade de troncos, caracterizam bosques mais complexos estruturalmente (Smith III, 1992). Observamos, ainda, que tais bosques possuem domínio de uma única espécie, como por exemplo $R$. mangle nas estações E11, E13 e F4 e L. racemosa nas estações E2, E3 e E10, caracterizando bosques praticamente monoespecíficos.

Outro fato que nos leva a concluir que esses manguezais foram alterados é a grande ocorrência de pontos com domínio de indivíduos de pequeno porte de $L$. racemosa, bosques estes que são típicos de locais alterados que se encontram em via de recomposição. Tal comportamento foi observado por Peria et al. (1990), referindo-se à dominância de L. racemosa, tanto em área basal, como em densidade de indivíduos, como característica de um processo de sucessão e de recomposição em bosques do Canal de Bertioga (SP), os quais, segundo Schaeffer-Novelli et al. (1990), são, normalmente, dominados por $R$. mangle. Tal processo também é descrito por Soares \& Tognella (1994) para manguezais do Canal de Bertioga. Smith III (1992) também relata que, em bosques de mangue sujeitos a maior freqüência de distúrbios, ocorre menor representatividade de espécies de Rhizophoraceae em comparação a espécies de outros grupos, como por exemplo o gênero Laguncularia.

A maior dificuldade no estudo das condições de conservação desses manguezais é a determinação tanto dos agentes degradadores do ecossistema na área da "Gleba E" como das condições de desenvolvimento do manguezal remanescente. Atividades relacionadas à movimentação de terra ou corte de manguezal seriam a causa mais provável da degradação dos bosques da "Gleba E", visto que, ao contrário da descarga de esgotos domésticos (segundo Zee, 1992, o principal problema ambiental das lagoas da Baixada de Jacarepaguá), constitui uma ação direta sobre a estrutura da vegetação, e que pode afetar a mesma de forma descontínua. Isso justificaria a existência de remanescentes de bosques mais desenvolvidos em alguns pontos da área estudada.

Assim, a variabilidade estrutural poderia estar relacionada a dois aspectos, o primeiro diria res- 
peito ao domínio de diferentes espécies em estações estruturalmente bem desenvolvidas, o qual estaria relacionado a características ambientais peculiares a cada estação, que seriam propícias para o maior desenvolvimento de determinada espécie (Clough, 1993). O segundo aspecto estaria relacionado à variabilidade no desenvolvimento estrutural, a qual seria indício de uma alteração relacionada a atividades antrópicas, visto que as características ambientais apresentadas por Zee (1993), tais como granulometria, salinidade e $\mathrm{pH}$, encontram-se de acordo com valores normais para o bom desenvolvimento de manguezais, independente das preferências de cada espécie (Brown \& Lugo, 1982; Cintrón \& Schaeffer-Novelli, 1983; Hutchings \& Saenger, 1987; Smith III, 1992; Aksornkoae, 1993; Cintrón-Molero, 1993; Clough, 1993; Ogino, 1993; Jimenez, 1994; Wakushima et al., 1994).

Esse aspecto ainda é corroborado, se notarmos que todas as estações estudadas estão relacionadas ao mesmo sistema, possuindo características similares, no que diz respeito à freqüência de inundação pelas marés, salinidade, aporte de água doce e de nutrientes, sem falar nas mesmas condições climáticas, o que propiciaria condições similares ao desenvolvimento estrutural.

Essa hipótese é reforçada quando levamos em consideração o atual processo de ocupação das margens da Lagoa da Tijuca, o que pôde ser comprovado através da análise de fotos aéreas tiradas em 1975 e 1993. Nesse período ocorreu a destruição total de alguns trechos de manguezal e a redução da faixa marginal de manguezal ao longo da área de estudo. Em 1975, a faixa de manguezal variava de 15 a $100 \mathrm{~m}$ de largura na região do Saco Grande e do Saquinho. Por ocasião dos trabalhos de campo (1993) foi constatada presença de uma faixa de manguezal que não ultrapassava os $40 \mathrm{~m}$ de largura, sendo que em alguns trechos essa vegetação era inexistente.

Por fim, podemos citar as observações de Zee (1993) que, através da análise de testemunhos geológicos realizados na área de estudo, constatou a presença de camadas de aterro na porção interna dos manguezais da "Gleba E", contribuindo para a redução da faixa de manguezal nessa área.

Em relação às estações localizadas na "Gleba F”, podemos constatar alterações significativas na estação $\mathrm{F} 4$, na qual encontramos (como já citado) bosques bastante desenvolvidos de $R$. mangle.
No entanto, o que chama atenção é a alta densidade de indivíduos de Acrostichum sp. e a quase inexistência de indivíduos jovens de espécies típicas de mangue. Através da análise dos testemunhos, Zee (op.cit.) nota a existência de uma camada espessa de lama praticamente sem matéria orgânica entre a camada superficial e uma camada sub-superficial, ambas com muita matéria orgânica de origem vegetal e raízes, tendo características de sedimento de manguezal, o que é confirmado por Roncarati \& Neves (1976).

A hipótese levantada é que o manguezal tenha sido alvo de sedimentação causada por algum episódio marcante, como por exemplo fortes chuvas, que tenham carreado grande quantidade de lama. Segundo Marques (1990), ocorre grande movimentação de massa (sedimentos) associada a fortes chuvas na região dos maciços da Tijuca e da Pedra Branca, cujo material mais fino tem como destino final as lagoas da Baixada de Jacarepaguá. Brandão (1992), analisando os índices pluviométricos para a região metropolitana do Rio de Janeiro num período de 140 anos (1851-1990), constata a ocorrência de chuvas anormalmente fortes com intervalos em torno de 10 a 11 anos.

Assim sendo, podemos sugerir que a sedimentação observada na "Gleba F" tenha sido provocada por eventos dessa magnitude, como por exemplo os ocorridos em fevereiro de 1988, quando três episódios pluviais isolados representaram, cada um, de $10 \%$ a $16 \%$ da pluviosidade média anual da cidade (Brandão, 1992). Tal fato é corroborado pelas observações realizadas na estação meteorológica do Aeroporto de Jacarepaguá, vizinho à área de estudo.

Os dados daquela estação indicam uma média anual para a precipitação de $1.116,4 \mathrm{~mm}$. No ano de 1988 foi registrada precipitação anual de 1467,7 $\mathrm{mm}$, sendo que apenas no mês de fevereiro foi atingido o valor de $378,0 \mathrm{~mm}$, quando a média desse mês, para a referida estação meteorológica, é de apenas 97,3 mm. Segundo Zee (1993), o domínio fluvial na área, durante o período de deposição da referida camada de lama, é comprovado ao se observar a ausência de carapaças de foraminíferos nesse sedimento.

Essa deposição não afetou tanto as árvores já existentes de mangue, mas provocou um levantamento da cota do terreno, diminuindo a influência das marés, propiciando a invasão por Acrostichum sp. Essa espécie teria impedido o desenvolvimento 
de indivíduos jovens de mangue. Tal fato pode vir a comprometer a existência do manguezal na área, visto que, como descrito por Cintrón \& SchaefferNovelli (1985), o bosque possui características de bosque maduro, em vias de alcançar um estágio de senescência (baixa densidade, árvores com dap $>20 \mathrm{~cm}$ e dossel pouco denso).

Dessa forma, a morte de um único indivíduo pode determinar a abertura de uma grande clareira (o que já ocorre no local), que em condições normais propiciaria o desenvolvimento dos jovens existentes no sub-bosque, os quais passariam a ocupar o espaço aberto no dossel (Smith III, 1992; Soares et al., 1993).

No entanto, devido a invasão de Acrostichum sp., isto não ocorre, não havendo assim a recomposição da área perdida com a morte das árvores adultas.

Apesar de não detectarmos uma mortalidade maciça dos manguezais da "Gleba E", devemos ter em mente que a redução da faixa marginal de manguezal, bem como a influência do aterro sobre o manguezal remanescente apresentada na análise dos testemunhos, identificando uma camada de aterro na parte interna dos manguezais da "Gleba E”, podem vir a constituir ações negativas crônicas sobre este manguezal, o que sem dúvida alguma viria a provocar o colapso deste ecossistema na área em questão.

Esse fato já é notado nas árvores localizadas na área de influência do projeto de ocupação, as quais apresentam indícios de estresse. Este processo é descrito por Lugo \& Snedaker (1974) e Lugo et al. (1980), ao detectarem alterações na complexidade estrutural de bosques de mangue afetados por aterro ou sedimentação intensa, bem como a morte dos mesmos e a conseqüente invasão por outras espécies.

Agradecimentos - Ao Professor David M. W. Zee do Departamento de Oceanografia, da Universidade do Estado do Rio de Janeiro, e sua equipe, pelo apoio nos trabalhos de campo. À Professora Dra. Yara Schaeffer-Novelli, do Instituto Oceanográfico da Universidade de São Paulo, pela leitura crítica do manuscrito.

\section{REFERÊNCIAS BIBLIOGRÁFICAS}

AKSORNKOAE, S., 1993, Ecology and management of mangroves. IUCN, Bangkok, Thailand, 176p.

ARAÚJO, D. S. D., 1978, As comunidades vegetais das margens das lagoas da baixada de Jacarepaguá. Cadernos FEEMA, Ser. Tec., 3/78, Rio de Janeiro, 35p.
BRANDÃO, A. M. P. M., 1992, As alterações climáticas na área metropolitana do Rio de Janeiro: uma provável influência do crescimento urbano, pp. 143-200. In: M. A. Abreu (ed.), Natureza e sociedade no Rio de Janeiro, Biblioteca Carioca, Secretaria Municipal de Cultura, Turismo e Esportes, Rio de Janeiro, 336p.

BROWN, S. \& LUGO, A. E., 1982, A comparison of structural and functional characteristics of saltwater and freshwater forested wetlands, pp.109-130. In: B. Gopal, R. E. Turner, R. W. Wetzel \& D. F. Whingham (eds.), Proceedings of the First International Wetlands Conference, Wetlands: Ecology and Management, New Delhi, India.

CEDAE, 1987, Disposição final dos esgotos sanitários da Baixada de Jacarepeguá. Rio de Janeiro, 140p.

CINTRÓN, G., LUGO, A. E., POOL, D. J. \& MORRIS, G., 1978, Mangroves of arid environments in Puerto Rico and adjacent islands. Biotrop., 10(2): 110-121.

CINTRÓN, G. \& SCHAEFFER-NOVELLI, Y., 1982, Mangrove forest - ecology and response to natural and man induced stressors. In: Workshop on coral reefs, seagrass beds and mangroves: their interactions in coastal zones of the Caribbean, St. Croix, U.S., Virgin Islands, Unesco Report in Marine Science, 23: 87-113.

CINTRÓN, G. \& SCHAEFFER-NOVELLI, Y., 1983, Introduccion a la ecologia del manglar. Oficina Regional de Ciencia y Tecnologia de la UNESCO para America Latina y el Caribe - ROSTLAC, Montevideo, Uruguay, 109p.

CINTRÓN, G. \& SCHAEFFER-NOVELLI, Y., 1984, Methods for studying mangrove structure, pp. 91-113. In: S. C. Snedaker \& J. G. Snedaker (eds.), The mangrove ecosystem: Research methods, UNESCO, Bungay, United Kingdom, 251p;

CINTRÓN, G. \& SCHAEFFER-NOVELLI, Y., 1985 , Caracteristicas y desarrollo estructural de los manglares de Norte e Sur America. Cienc. Interam., 25(1-4): 4-15.

CINTRÓN, G., LUGO, A. E. \& MARTINEZ, R., 1985, Structural and functional properties of mangrove forests, $\mathrm{pp}$. 53-66. In: W. G. D'Arcy \& M. D. A. Correa (eds.), The botany and natural history of Panama, Missouri Botanical Garden, Saint Louis, Missouri.

CINTRÓN-MOLERO, G., 1993, Mangroves of arid regions of Puerto Rico and the Caribbean, pp. 117-122. In: H. Lieth \& A. Al Masoom (eds.), Towards the rational use of high salinity tolerant plants, v. 1, Kluwer Academic Publishers, The Netherlands, 521p.

CINTRÓN-MOLERO, G. \& SCHAEFFER-NOVELLI, Y., 1992, Ecology and management of New World mangroves, pp. 233-258. In: U. Seeliger (ed.), Coastal plant communities of Latin America, Academic Press, California, 392p.

CLOUGH, B. F., 1993, Constraints on the growth, propagation and utilization of mangroves in arid regions, pp. 341-352. In: H. Lieth \& A. Al Masoom (eds.), Towards the rational use of high salinity tolerant plants, v. 1, Kluwer Academic Publishers, The Netherlands, 521p.

HUTCHINGS, P. A. \& SAENGER, P., 1987, Ecology of mangroves. University of Queensland Press, Brisbane, $388 \mathrm{p}$. 
JIMENEZ, J. A., 1994, Los manglares del Pacifico centroamericano. C. R. EFUNA, Heredia, 352p.

JIMENEZ, J. A., LUGO, A. E. \& CINTRON, G., 1985, Tree mortality in mangrove forests. Biotrop., 17(3): 177-185.

LUGO, A. E., 1990, Fringe wetlands, pp. 143-169. In: A. E. Lugo, M. Brinson \& S. Brown (eds.), Forested wetlands. Ecosystems of the world, Elsevier, Amsterdam, 527p.

LUGO, A. E., BRINSON, M. M. \& BROWN, S., 1990a, Synthesis and search for paradigms in wetland ecology, pp. 447-460. In: A. E. Lugo, M. Brinson \& S. Brown (eds.), Forested wetlands. Ecosystems of the world, Elsevier, Amsterdam, 527p.

LUGO, A. E., BROWN, S. \& BRINSON, M. M., 1990b, Concepts in wetland ecology, pp. 53-85. In: A. E. Lugo, M. Brinson \& S. Brown (eds.), Forested wetlands. Ecosystems of the world, Elsevier, Amsterdam, 527p.

LUGO, A. E., CINTRÓN, G. \& GOENAGA, C., 1980, El ecossistema del manglar bajo tension, pp. 261-285. In: Seminario sobre el estudio cientifico e impacto humano en el ecossistema de manglares, Cali, Colombia, UNESCO/ROSTLAC.

LUGO, A. E. \& SNEDAKER, S. C., 1973, Properties of a mangrove forest in south Florida, pp. B1-B87. In: S. C. Snedaker \& A. E. Lugo (eds.), The role of mangrove ecosystems in the maintenance of environmental quality and high productivity of desirable fisheries, Bureau of Sport Fisheries and Wildlife, Center for Aquatic Sciences, University of Florida.

LUGO, A. E. \& SNEDAKER, S. C., 1974, The ecology of mangroves. Ann. Rev. Ecol. Syst., 5: 39-64.

LUGO, A. E. \& ZUCCA, C. D., 1977, The impact of low temperature stress on mangrove structure and growth. Trop. Ecol., 18: 149-161.

MARQUES, J. S., 1990, A participação dos rios no processo de sedimentação da Baixada de Jacarepaguá. Tese de Doutorado, UNESP, Rio Claro, SP, 435p.

OGINO, K., 1993, Mangrove ecosystem as soil, water and plant interactive system, pp. 135-143. In: H. Lieth \& A. Al Masoom (eds.), Towards the rational use of high salinity tolerant plants, v. 1, Kluwer Academic Publishers, The Netherlands, 521p.

PERIA, L. C. S., FERNANDES, P. P. C. P., MENEZES, G V., GRASSO, M. \& TOGNELLA, M. M. P., 1990, Estudos estruturais comparativos entre bosques de mangue impactados (Canal de Bertioga) e não-impactados (Ilha do Cardoso), Estado de São Paulo, pp. 183-193. In: Anais do II Simpósio de Ecossistemas da Costa Sul e Sudeste Brasileira: estrutura, função e manejo, Águas de Lindóia, São Paulo, Academia de Ciências do Estado de São Paulo, v. 2.

RONCARATI, H. \& NEVES, L. E., 1976, Projeto Jacarepaguá. Estudo geológico preliminar dos sedimentos recentes superficiais da Baixada de Jacarepaguá, Município do Rio de Janeiro. PETROBRÁS/CENPES - DEXPRO, Rio de Janeiro, 89p.

SCHAEFFER-NOVELLI, Y. \& CINTRÓN, G., 1986, Guia para estudo de áreas de manguezal. Estrutura, função e flora. Caribbean Ecological Research, São Paulo, 150p.
SCHAEFFER-NOVELLI, Y., CINTRÓN-MOLERO, G. \& ADAIME, R. R., 1990, Variability of mangrove ecosystems along the brazilian coast. Estuar., 13(2): 204-218.

SILVA, C. A. R., LACERDA, L. D., SILVA, L. F. F. \& REZENDE, C. E., 1991, Forest structure and biomass distribution in a red mangrove stand in Sepetiba Bay, Rio de Janeiro. Rev. Brasil. Biol., 14:21-25.

SMITH III, T. J., 1992, Forest Structure, pp.101-136. In: A. I. Robertson \& D. M. Alongi (eds.), Coastal and Estuarine Studies, v. 41, Tropical mangrove ecosystems, American Geophysical Union, Washington, D.C., 329p.

SOARES, M. L. G., PERIA, L. C. S. \& SCHAEFFERNOVELLI, Y., 1993, Preliminary study on the dynamics of Rhizophora mangle seedlings and saplings in a mangrove stand at Cardoso Island, São Paulo, Brazil, p.170. In: Asia-Pacific Symposium on Mangrove Ecosystems, The Hong Kong University of Science \& Technology, Abstracts.

SOARES, M. L. G. \& TOGNELLA, M. M. P., 1994, Diagnóstico ambiental dos manguezais próximos ao empreendimento Marina Guarujá, Guarujá, São Paulo. Relatório não-publicado, $11 \mathrm{p}$.

STRANG, H. E., CASTEllanos, A., CHAVES, C. M., ATALA, F., MARTINS, H. F., COIMBRA-FILHO, A. F., SOARES, I. L. \& CAMPOS, T. T., 1965, A restinga de Jacarepaguá. Bol. Geogr., 187: 538-546.

TWILLEY, R. R., 1995, Properties of mangrove ecosystems related to the energy signature of coastal environments, pp. 43-61. In: C. Hall (ed.), Maximum power, University of Colorado Press, Boulder, Colorado.

WAKUSHIMA, S., KURAISHI, S. \& SAKURAI, N., 1994, Soil salinity and $\mathrm{pH}$ in japanese mangrove forests and growth of cultivated mangrove plants in different soil conditions. J. Plant Res., 107: 39-46.

ZEE, D. M. W. (ed.), 1992, Estudo ambiental: poluição dos recursos hídricos da Baixada de Jacarepaguá. Relatório não-publicado, Universidade do Estado do Rio de Janeiro, 75p.

ZEE, D. M. W. (ed.), 1993, Estudo ambiental: faixa marginal da Lagoa da Tijuca, RJ. Relatório não-publicado, Universidade do Estado do Rio de Janeiro, 83p. 